Contributions from the United States National Herbarium) entnehme ich, daß dieser bereits die generische Selbständigkeit von Pisoniella erkannte, weshalb die Bezeichnung Pisoniella arborescens (Lag. et Rodr.) Standley zu gelten hat.

\title{
Die Stammpflanze des offizinellen Rhabarbers und die geographische Verbreitung der Rheum-Arten.
}

Von Dr. Carl Curt Hossens (Berchtesgaden).

Die äußere Veranlassung, mich mit der Rhabarberfrage näher zu beschäftigen, wurde dadurch gegeben, daß Herr Dr. Alber Tafel, der bekannte Tibetforscher, mir nach seiner Rückkehr seine äußerst interessante botanische Ausbeute zur Bestimmung übergab. Trotzdem leider ein großer Teil der Sammlung infolge der verschiedensten Unglücksfälle verloren ging, sind doch noch außer einer großen Anzahl von Moosen und Flechten - insgesamt ca. 330 Nummern von Phanerogamen vorhanden, unter denen

auch fünf Rhabarberpflanzen befinden. Vorliegende Arbeit wurde bereits im Jahre 1909 im Botanischen Museum zu Dahlem bei Berlin begonnen, konnte aber erst 1911 im Kew Herbarium vollendet werden. Infolge der Bedeutung der Rhabarberfrage für die Medizin habe ich mich entschlossen, die rten von Kew, sowie solche ron St. Petersburg und Berlin zu $v$ gleichen und dieses gesamte Material zu verwenden.

Im folgenden beabsichtige ich, zuers die historische Frage der Einführung des Rhabarbers, im Anschluß daran kurz die medizinische Bedeutung zu erörtern, dann bauptsächlich auf die Zwe ckmäßigkeit der Anpflanzung von Rheum palmatum für Deutschland und Österreich-Ungarn hinzuweisen und im Anschlusse daran die von mir besichtigten anderen Arten pflanzengeographisch zusammenzufassen.

Marco Polo und die gelehrten Jesuiten, die im Auftrage der portugiesischen Regierung $\mathrm{Ch}$ in a bereisten und kartographisch aufnahmen, waren vermutlich die ersten Europäer, die den echten Rhabarber gesehen haben. Leider haben sie aber weder Pflanzen noch Samen gesammelt, noch eine wissenschaftliche Beschreibung der damals als Stammpfianze angenommenen Rheum-Art gemacht. Sie alle stimmen nur darin überein, daß sie aus dem Lande der "Tanguten" komme. Nebenbei sei hier erwähnt, daß der Name "Tanguten" nach $\mathrm{Tafel}^{1}$ ) zu unrecht besteht. "In Osttibet wohnt,

1) A. Ta fel im Korrespondenzblatt der Deutschen Gesellschaft für Anthropologie, Ethnologie und Urgeschichte, XXXIX. Jahrg., Nr. 9/12, Sept./Dez. 1908. 
rom See Kuku-nor im Norden bis an die Himalayaketten im Süden ein Volk, das tibetisch spricht. Eine Trennung in "Tanguten“ und "Tibeter" hat keine Berechtigung. Das Wort "Tangut" ist nur irreführend und sollte womöglich vermieden werden. Es ist uns damit ähnlich ergangen, wie einst mit dem Namen „Katay" und "China". Die Reisenden, die von Norden kamen und deshalb Mongolen um Rat fragten, erfuhren, daß die Bewohner "Tangutse* hießen. "Dies ist einfach die Bezeichnung für die Tibeter im allgemeinen. Die Reisenden, die von Süden kamen, hörten und lasen gleich von Anfang an den tibetischen Namen "Bod"..."

Das größte Interesse an der Lösung der Frage nach der Stammpflanze hatte Rußland, das in innigem Verkehr mit China stand und den gesamten Transitrerkehr nach dem Westen Europas vermittelte. So kam es denn, daß nach C. J. Maximovicz ${ }^{1}$ ) bereits früh im 18. Jahrhundert im sibirisch-chinesischen Grenzorte Kjachta eine Rhabarber- „Brake“ (Kontrollstation) eingerichtet wurde, wo aller von China importierte Rhabarber einer strengen Sichtung unterworfen wurde. Die Folge hiervon war, da\& dieser sog. muskovitische Rhabarber als der beste auf den westeuropäischen Märkten galt.

Die Versuche von seiten Rußlands, Samen der Stammpflanze zu erbalten, brachte im Jahre 1740 die erste Verwirrung in die Rhabarberfrage. Ließ sich doch selbst Linné verführen, die aus den chinesischen Samen gezüchteten Pflanzen als Rheum Rharbarum zu beschreiben, einen Namen, den er selbst bald einzog und in Rh. undulatum änderte. Ich führe nun Maximovi $\mathrm{cz}^{2}$ ) wörtlich an: "Allein ums Jahr 1750 wurden neve Samen erworben und diesesmal erwuchs in der Tat das echte Rheum palmatum, eine noch nie gesehene Art, daraus hervor. So war denn die echte Rhabarberpflanze, dauk den Bemühungen Rußlands, für Earopa gewonnen, fand rasche Verbreitung und wurde in den Achtzigerjahren des vorigen Jabrhunderts in Schottland, England, Deutschland bereits im großen kultiviert, ja in den zwei erstgeuannten Ländern, wo die Gartenbaugesellschaften ihre Kultur und Zubereitung auf jede Weise aufmunterten, wurde die Wurzel bereits zu einem Handelsartikel, von manchen Ärzten der chinesischen an Wirkung vollkommen gleich befunden und in der Praxis ausschließlich gebraucht. Es ergab sich aber aus den zahlreichen Versuchen, daß nur die alte (etwa mindestens achtjährige) Wurzel eine gute Drogue liefere, daß aber auch selbst bei dieser sehr viel auf die Zeit der Ernte, die nachmalige Behandlung und vor allem das Trocknen ankomme, daß ferner die Stammwurzel und nicht die Äste derselben die kräftigste Arznei lieferten, daß aber gerade die erstere leicht ausfaule und deshalb die Kultur eine

1) C. J. Maximowicz in Regels Gartenflora, Jänner 1875, pag. 3-10. 2) 1. c., pag. 4. 
schwierige sei $\left.{ }^{1}\right) \ldots$ Dennoch hätte sich allmählich Rheum palmatum seinen Weg gebahnt, wenn nicht bald, gerade von Rußland aus, Zweifel geäußert worden wären, daß dies doch nicht die Stammpflanze des echten Rhabarbers sei."

Und jetzt beginnt das Schauspiel von neuem, das sich bis in die jüngste Vergangenheit hinzog ${ }^{2}$ ). Pallas und Sievers wurden von den Chinesen getäuscht, indem diese erklärten, die in Europa getrockneten Rheum-Blätter stammen nicht von der echten Art; diese habe ungeteilte Blätter. Die Engländer gaben eine indische Form des Himalaya, $R h$. australe, als die Rhabarberpflanze aus, bis General - damals noch Oberstleutnant - Przewalski in den Jahren 1871 bis 1873 die echte Art, wiederum Rheum palmatum L., in "Kansu“ auf dem „Bergland am den Kuku-nor", also in Tibet, antraf und damit definitiv wenigstens für diese Zeit die Frage zugunsten des guten, alten Rheum palmatum L. entschied.

Es sei nun gestattet, hier im Wortlaut anzuführen, was Maximowicz ${ }^{3}$ ) angibt, von Przewalski erfahren zu haben:

"Der echte Rhabarber, bei den Mongolen Schara moto (Gelbholz), bei den Tanguten D shumza genannt, wurde von ihm zuerst beobachtet in den Gebirgen am Mittellanfe des Flusses Tetung-gol, wo er übrigens nicht sehr häufig zu nennen ist. Dagegen soll er in außerordentlicher Menge, nach Aussage der Tanguten, am Oberlaufe desselben Flusses und des Entsine wachsen, wo auch die Hauptmasse der Wurzeln gegraben wird. Ferner wurde Rheum palmatum in Menge angetroffen in den Waldgebirgen bei der Stadt Sinin, südlich vom See Kuku-nor in den Gebirgen, sowie in der Bergkette Jegrai-ula in der Nähe der HoanghoQuellen. Dagegen fehlt die Pflanze in den Bergen des nordöstlichen 'libet ganz, vielleicht wegen der Waldlosigkeit derselben. So ist denn ihr Verbreitungsgebiet auf das Bergland um den Kuku-nor beschränkt. Ob sie ostwärts in der gebirgigen Provinz Szetschwan vorkomme, konnte Przewalski nicht erfahren."

Auch der bedeutendste Chinaforscher, Ferdinand Freiherr v. Richth of en, gibt jenes Gebiet als den Standort für den echten Rhabarber an, ohne sich auf die Frage nach-der Stammpflanze weiter einzulassen.

Farre bezeichnet direkt Rheum palmatum als solches für den besten chinesischen Rhabarber.

Wieder und wieder suchte aber der eine oder andere Reisende vor allem aus dem Himalayagebiet sich durch die Mitteilung wichtig zu machen, er babe endlich (!) die echte Rhabarberpflanze gefunden; so kamen denn eine größere Anzahl neuerer Arten, die

1) Im „Archiv für Pharmazie“, p. 423 (1911), babe ich bereits darauf hingewiesen, "daL die Erfahrung in den Kew Gardens dahin geht, daß die im Schatten und Halbschatten stehenden Pflanzen ausgezeichnet gedeihen, im Gegensatz zu den in direkter Sonnenbestrahlung stehenden.

2) Vergl. auch H. Zö rnig, „Arzneidrogen“.

3) .C. J. Maximowicz, l. c., pag. 6.

Osterr. botan. Zeitsehrift. 12. Heft. 1911. 
zum T'eil mehr oder weniger ausgesprochene Bastarde waren, nach Europa. Die schwerste Konkurrenz erbielt Rheum palmatum durch das von Baillon beschriebene Rheum officinale. Auch der Verteidiger des ersteren, Tschirch, fiel um, ja sogar entgegen seinen eigenen chemischen Beobachtungen: „Endlich zeigt sich, daß vo n allen in Bern kultivierten Rheum-Arten unzweifelhaft Rh. palmatum die höchstprozentigen Rhizome liefert $(2 \cdot 8 \%)$, während $R h$. officinale (2\%) und Polinianum $(1 \cdot 8 \%)$ ihm weit nachstehen", als Wils on 1906 die Angabe machte: "Rhabarber wird nur von einer Art gesammelt und diese Art ist Rheum officinale." Daran schließen sich wieder Gründe und Hypothesen an. Obwohl nun Tschirch damals schon Samen von Tafel hatte, faßt er seine Ansicht doch dahin zusammen:

${ }_{n}$ So läge denn die Sache so, daß der "südliche" Rhabarber aus Szetschwan von Rheum officinale, der „ürdliche $\mathrm{e}^{*}$ om Kuku-nor von Rheum palmatum $\beta$. tanguticum stammt", wie wir bisher schon auch in Englers Syllabus lasen und lernten!

Tafel stellt nun ausdrücklich, ebenso wie Przewalski, Richthofen, Farre, früher schon die gelehrten Jesuiten und mancher andere, fest, daß der echte, gute Schensi-Rhabarber, also die medizinisch wertvolle Droge aus Tibet von Rheum palmatum L. stammt.

Erfreulicherweise hat Mr. Wils on ebenfalls seine frühere Ansicht einstweilen modifiziert. Wie veröffentlicht, schrieb er mir: "The medicinal rhubarb collected around Tatien-lu (Tachien-lu) and also in Western Hupeh is most probably $R$. officinale as stated in 1906. That collected around Sungpan, North-Western Szechuan, and in the Tibetan country to the west and north-west of that region, is unquestionably $R$. palmatum, var. tanguticum."

Diese Aussage von heute lautet ganz anders als früher: Für Rheum officinale nur noch ein zagendes "most probably" und für Theum palmatum ein sicheres "unquestionably“.

Also auch hier hat Tafel indirekt den Stein ins Rollen gebracht; schlie $3 t$ doch Wilson mit dem alle Zweifel nehmenden und für die Praxis einzig und allein wichtigen Satz:

"The rhubarb from the Sungpan regions (R. palmatum var. tanguticum) is considered by the (hinese to be much superior to the rbubarb from the Tatien-lu ( $R$. officinale) and it fetehes a considerably higher price in the market."

Wir wollen uns jetzt der. Gewinuung der Wurzeln oder Rhizome zuwenden. Maximowicz führt hierzu nach Przewalski's Mitteilungen aus: „Die Wurzel hat eine länglich abgerundete Form und gibt bis zu "25 Seitenäste von 12-21 Zoll Länge und $1 \%$ Zoll Dicke ab. Ihre Größe hängt vom Alter ab und kann für die Hauptwurzel 1 Fuß in Länge und Dicke erreichen. $\nabla$ on auß en ist sie mit einer bräunlichen rahen Rinde bekleidet, welche beim Trocknen der Wurzel mit dem Messer ent- 
fernt wird.... Die dicken Wurzeln werden in mebrere Stücke, die stärkeren Seitenäste gleichfalls in etliche Querstücke geschnitten, diese Stücke auf Schnüre gereiht und an einem schattigen Ort (gewöhnlich unter das Dach des Hauses) zum Trocknen aufgehängt. Die Chinesen kaufen die so zubereitete Wurzel auf....".

Interessant ist hier wieder die Ansicht von Professor Tschirch $\left.{ }^{1}\right)$ :

„Wenu der Rhabarber getrocknet werden soll, werden vor allen Dingen die Wurzeln abgeschnitten, dann die dunkelbraune "Rinde“ abgeschnitten, das Rhizom gespalten und auf Fäden gezogen an einem schattigen, luftigen Orte, z. B. unter dem Dache des Hauses aufgehängt, also nicht in der Sonne - trotzdem im Handel „sun dried“ genannt - und nicht mit künstlicher Wärme getrocknet. Der Handel unterscheidet jetzt aber auch „high dried“, d. h. künstlich getrocknete. Auch Chauvean berichtete bereits 1874, daß bisweilen künstliche Wärme (bei schlechten Sorten) angewendet werde.

Die dickeren Seitenwurzeln werden übrigens nicht weggeworfen, sondern wie das Rhizom behandelt und mit diesem verkauft. Sie gelangen aber niemals nach Europa. Ich habe mehrere Tausend Stück Rhabarber verschiedenster Provenienz genau angesehen, aber niemals eine Wurzel gefunden (!!).

Wann das Schälen erfolgt, ob an dem frischen Rhizom oder am halbgetrockneten, oder am trockenen, wird nirgends angegeben. Nach den hier in Bern an kultivierten Rhizomen gesammelten Erfahrungen möchte ich die bestimmte Ansicht aussprechen, daß das frische Rhizom wahrscheinlich nur oberflächlich geschält wird, das eigentliche "Mundieren“ erst am halbgetrockneten oder ganz trockenen erfolgt. Darauf deutet auch eine Bemerkung von Farre, daß nach dem Graben nur die schwarze Haut entfernt werde, "the black skin with covers it is removed."

Hat Herr Professor Tschirch nicht mehr gelesen, was Maximowicz weiter schreibt? "Diesen Mitteilungen des Herrn Przewalski kann ich noch hinzufügen, daß einige Pud trockener Wurzeln, welche dieser Reisende mitbrachte...." und "Der einzige rein äußerliche Unterschied zwischen ihnen und der früberen Kjachta-Ware besteht darin, daß sie nicht wie diese einer zweiten Reinigung und Sichtung unterworfen gewesen waren, daher ihnen noch kleine Schichten Rinde anhaften und die Löcher, durch welche die Schnüre liefen, an welchen sie aufgehängt gewesen waren, kleiner im Durchmesser sind, während in der Kjachta-Brake alle Rinde sorgfältig abgeraspelt und die Löcher ausgeweitet wurden, um alle entfürbten oder schlechten Teile zu entfernen."

i) A. Tschirch, "Studien über den Rhabarber und seine Stammpflanze“, Wien 1904, 1. pag. 68 . 
Ich glaube, Tschirch hat diese Sätze gelesen, denn er fährt später weiter: "Przewalski brachte nun von seiner Reise sowobl Samen, wie trockene Pflanzen vom Kuku-nor mit.“

Auf die Frage des Trocknens habe ich bereits im „Archiv für P harmazie" hingewiesen und dort ausgeführt: „Hiezu möchte ich bemerken, daß "high dried" wohl besser mit "hoch oder bängend getr ocknet" zu übersetzen ist und nichts mit "künstlicher Wärme“ zu tun hat. Außerdem bat die Bezeichnung "sun dried" auch ihre Berechtigung. Bei Dr. Tafel finden wir nämlich folgende Sätze: „Die Zedernwälder Ost-Ts'aidams sind die wahre Heimat der besten Sorte unseres in den Apotheken verwerteten sogenannten Schensi-Rhabarbers. In den Monaten August und September kommen zahlreiche chinesische Mohammedaner dorthin und gehen im Raubbau den Rhizomen nach, die an Ort und Stelle geschält und getrocknet werden." Auf der Etikette der Zweige ist angegeben, das die Rhizome an Stricken von einem Baume zum anderen, di $e$ nicht sehr dieht stehen, getrocknet werden.

Wir sehen hieraus, im Gegensatz zur Ansicht von Przewalski, daß die Rhizome fast immer im Freien und unter Bäumen getrocknet werden; das Trocknen in den Häusern ist selten. Diese sind dann als "high dried" in den Handel gebracht, während erstere unter den "wenig Schatten spendenden" Zedern getrockneten Rhizome als "sun dried" bezeichnet werden.

Von Interesse sind die sich widersprechenden Angaben von Przewalski nach Maximowicz und ron Przewalski nach Tschirch über die von den Tibetern angeptlanzten Rheum-Stöcke: "In geringem Quantum und ausschließlich zu eigenem Gebrauche als Arznei für Menschen und Vieh wird der Rhabarber von den Tanguten auch in ihren Gemüsegärten gezogen, wozu man ihn entweder säet oder die jungen Pfänzchen im Walde aufsucht und ausgräbt. Die Aussaat kann im Frühjahr wie im Herbst geschehen, aber soll die Kultur Erfolg haben, so ist stets reiner, lockerer, feinkörniger und feuchter schwarzer Humus das unumgängliche Erfordernis. Die Tanguten sagen, im dritten Jahre nach der Aussaat erreiche die Hauptwurzel Faustgröße, erlange aber die erforderlichen Maße erst acht, zehn und mehr Jahre nach der Aussaat. Übrigens soll, nach Aussage der'Tanguten, der kaltivierte Rhabarber in seinen Eigenschaften dem wildwachsenden durchaus entsprechen."

Tschirch schreibt: "Die Tanguten kultivieren auch etwas Rhabarber, der kultivierte ist aber nichts wert und wird nur für das Vieh und in der heimischen Medizin verwendet." Das ist gerade das Gegenteil von den Angaben Przewalski's!

Wir können diesen Satz auch deshalb nicht unwidersprochen lassen, weil er gerade die Absicht, Rheum palmatum in Europa im Freien zu züchten, als völlig aussichtslos hinstellt und der sonst vertretenen Tendenz deshalb widerspricht. Gerade weil 
die Tibeter aus Rheum palmatum gute Rhizome, wenn auch erst nach längerem Warten, erzielten, können und müsen wir diese im Gegensatz zu Rheum officinale $\mathrm{zu}$ Versuchen im großen anempfehlen. Tschirch kommt auf Grund seiner chemischen Analysen zu der Ansicht: " daß alle guten chinesischen Rhabarberrhizome nicht älter als höchstens vier Jahre sind", da sie fünfjährig, weich und schwammig oder hart und holzig, dabei arm an Oxymethylanthrachinomen werden. Wie wir sehen werden, hängen diese Mißstände mit unrichtigen Pflanzungsmethoden zusammen.

Für die Kultur möchte ich wieder Maxi mow i cz das Wort geben : „Die Regeln für die Kultur des Rhabarbers (Rh. palmatum L.) ergeben sich nach all dem Gesagteu von selbst: ein leichter, lockerer, tiefer, schwarzer Humus [wie er z. B. in dem Berchtesgadener Land in Bayern oder in den Seitentälern der Julischen Alpen so vortrefflich ist]; Anpflanzen in solchen Zwischenräumen, daß jedes Individiduum Raum zur vollständigen Entwicklung hat (also etwa $8 \mathrm{Fu}$, damit die Blätter sich ordentlich auslegen können); Beschattung durch Bäume, regelmäßiges Begießen (weil Kansu ein feuchtes Klima hat) [also auch hier wäre die Gegend Salzburg, Berchtesgaden, Bad Reichenhall hervorragend!] und Exposition nach Süden. - AuGerdem glaube ich auf zweierlei aufmerksam machen zu müssen.

Nach Analysen des (salzigen) Kuku-nor-Wassers, welche Prof. Schmidt in Dorpat an von Przewalski mitgebrachten Proben ausgeführt hat, ist dieses Wasser viel reicher an Kalksalzen als andere Gewässer, und Prof. Schmidt schreibt dies kalkhaltigen Süßswässern zu, die den See speisen. $\mathrm{Da}$ nun der Gehalt der Rhabarber-Wurzel an medizinisch wirksamen Bestandteilen Hand in Hand geht mit ihrem Gehalt an Kristallen von oxalsaurem Kalk, so daß eine gute Wurzel sogleich erkannt wird, wenn sie beim Kauen stark unter den Zähnen knirscht, so scheint hartes, kalkhaltiges Wasser für dieselbe Bedürfnis zu sein, damit die Pflanze demselben den ihr notwendigen Kalk zur Kristallbildung entuehmen könne. Vielleicht ist allein das Nichtbeachtendieses Erfordernisses daran schuld, daß die früher in Europa liultivierte Wurzel von $R h$. palmatum, so ähnlich siesonst der chinesischen war, nichtunter den Zähnen knirschte und weniger kräftig in ihrer Wirkung war."

Zuletzt weist Masimowiez darauf hin, daß sich das Regenwasser in den Höhlungen ansammelt und so eine immer tiefer hineinfressende Fäule hervorruft [wie ich dies auch bei den in der Sonne stehenden Exemplaren in Kew Gardens beobachten konnte, von denen im Frühling von 21 Blätter 13 am Verfaulen waren, während im August Rheum palmatum a. typicum zumeist schlechte Blätter und der als $\beta$. tanguticum bezeichnete Rhabarber nur mehr. füuf verkämmerte Blätter in der Sonve aufwies]. „Wie dies am besten zu verhindern wäre, muß weiteren Versuchen zu entscheiden über- 
lassen werden: Wegputzen der alten Blattscheiden, frühzeitiges $A b$ schneiden der verblühten Stengel, Bedecken oder Verschmieren der Stengelnarben scheinen am leichtesten ausführbar. Am besten wäre vielleicht, man ließe die Pflanze überhaupt nicht zum Blühen kommen."

Der letztere Vorschlag ist sicher ganz vortreff lich.

Betrachten wir nun die Anforderungen, die an die Kultur von Rheum palmatum gestellt werden, so sehen wir, daß diese z. B. in Bayern am besten in den regenreichen Kalkalpen, also speziell dem Gebiet von Bad Reichenhall und Berchtesgaden, in Osterreich in dem von Salzburg, den julischen Alpen oder Karawanken in hervorragendem Maße erfüllt würden; ebenso würden sich wohl Strecken in Dalmatien und Istrien vortrefflich zu größeren Versuchen eignen.

Wir wollen außerdem nicht vergessen, daß die Stiele von Rheum palmatum nicht nur in der asiatischen, sondern auch in der europäischen Küche, so noch heute in England, eine große Rolle als Nahrungsmittel spielen. Hat man die Gebirgsbevölkerung einmal daran gewöhnt, so wird sich rasch dafür ein guter Absatz finden, um so mehr als auch in diesen wenigstens etwas von dem medizinischen Nutzen der Rhizome und Wurzeln vorhanden ist.

Was nun die medizinische Bedeutung des Rhabarbers anbelangt, so verdanke ich meinem Freunde Dr. med. Carl H ofmann (Berlin) und Professor Lewin (Berlin) eine Anzahl wertvoller Mitteilungen, deren Angaben aber auf mir fremde Gebiete führen würden. Hier nur so viel, daß infolge der Stoffe Chrysoptensäure und Rheum-Gerbsäure der Rhabarber eine doppelte Wirkung hat: in kleinen Dosen $(0.1-0.5)$ wirkt er entweder gar nicht oder appetitanregend und antidiarrhitisch; in größeren Dosen $(1 \cdot 0-5 \cdot 0)$ dagegen als mildes Abführmittel.

(SchluB folgt.)

\section{Conioselinum tataricum, neu für die Flora der Alpen.}

Von Friedrich Vierhapper (Wien).

(Mit 2 Textabbildungen und 1 Verbreitungskarte.)

(Fortsetzung. ${ }^{1}$ )

Unsere Annahme, daß die subarktisch-subalpine Artgenossenschaft erst am Ausgange des Pliozän und ebensowenig früher als später nach Mitteleuropa eingewandert ist, findet auch durch die Berücksichtigung der hentigen Verbreitung und der Verwandtschaftsverhältnisse ihrer Arten eine wesentliche Stütze. Insbesondere zwei Umstände erscheinen mir erwähnenswert.

1. Die meisten der Arten haben in den mitteleuropäischen -Gebirgen mehr oder minder zerstückelte Areale und machen hier

1) Vgl. Nr. 11, S. 435. 